\title{
Performance and Operational Experience of the LEP Synchrotron Light Telescopes
}

\author{
G. Burtin, R.J. Colchester, J.J. Gras, R. Jung, J.M. Vouillot \\ European Organization for Nuclear Research (CERN) \\ CH-1211 Geneva 23, Switzerland
}

\section{Abstract}

The experience and performance over the past three and a half years of operation is reported. The dynamic range of the monitor has been increased by an improved cooling of the CCD chip with the help of Peltier cells. The contributions from the diffraction and the depth of field have been evaluated with precision in order to estimate the beam emittances. Comparisons and cross calibrations with the wire scanners have been started. A video frame grabber and fast projection calculation for real time beam size display has been implemented.

\section{INTRODUCTION}

There are four synchrotron radiation (SR) telescopes located around Intersection 8 of LEP. The system has been described in detail in [1]. The monitors have been in operation since the start-up in July 1989. Two monitors observe the electron beam and two the positron beam. For each type of particle, there is a monitor located at a near zerodispersion location and one at a high dispersion point. The telescopes have a magnification of $1 / 5$ between the beam and the detectors. Two detectors per telescope are in use. The first and most used one is a CCD chip which integrates the light coming from all bunches over a maximum period of $20 \mathrm{~ms}$. The second is a gated intensificr and wavelength shifter coupled to a CCD which is able to acquire individual bunches at selected intervals. The effort over the past year was mainly directed towards the improvement of the precision on emittance measurements. For this, the dynamic range was increased and the contributions of the depth of field and diffraction were measured with better precision.

\section{IMPROVEMENT OF THE DYNAMIC RANGE}

The dynamic range of the monitor is first limited by that of the CCD chip. Charges of thermal origin, the so-called dark current, are collected in the CCD potential wells during the integration and readout periods. They decrease the dynamic range, introduce statistical non-uniformities on the signals and a slope on the vertical beam projection. The latter is generated by the difference in time spent in the CCD by the various pixel charges as a function of their origin. The dark current can be reduced by a shorter readout time and by cooling the $C C D$ chip. For best geometrical precision, the CCD chip, of the frame transfer type, is read out pixel by pixel at a frequency up to $1 \mathrm{MHz}$, limited by the speed of the 12 bit ADC and the $500 \mathrm{~m}$ cable length from the camera head to the processing electronics. This readout can take up to 200 ms. A faster readout frequency would decrease the dark current but also increase the noise of the output stage and the charges lost during the many transfers to the output.
Experiments have been carried out at various temperatures of the CCD chip, at read-out frequencies of $450 \mathrm{kHz}, 900 \mathrm{kHz}$ and $7 \mathrm{MHz}$, in full darkness and with uniform illumination, in order to find optimum operating conditions. The CCD temperature was changed by connecting to its back face a Peltier effect cooling cell and by providing a cooled dry air circulation in the telescope. The chip temperature was lowered, starting from an estimated temperature of $60^{\circ} \mathrm{C}$ with the original closed camera [1]. The slope of the vertical projection was reduced from one hundred counts (out of 4096) to one count over the 288 useful lines as soon as a temperature below $15^{\circ} \mathrm{C}$ was reached. The noise of the two projections was FFT analysed. The dominant noise is the quantification noise of the 12 bit ADC both in darkness and with a uniform illumination. This justifies the use of an ADC of at least 12 bits if the conversion time is not a major issue. Switching from 450 to $900 \mathrm{kHz}$ gave little improvement. The Dark Current Non Uniformity and the Photon Response Non Uniformity were estimated to be in the range of $+/-1$ count and the detector was found to be linear over 3500 counts. The charges lost during the numerous transfers to the output amplifier are characterised by the Charge Transfer Efficiency (CTE). At $450 \mathrm{kHz}$ and near saturation, a CTE slope of $-2.10^{-4} /$ pixel has been measured. It results in a maximum charge loss of $20 \%$. This slope increases with frequency. As a consequence, all CCD cameras will be of the open frame type with one Peltier cooling cell and will be read at a frequency of $450 \mathrm{kHz}$, except for one detector which will be operated at $900 \mathrm{kHz}$ for test purposes. Good projections are obtained in practice at a signal peak amplitude of one hundred counts. With the available neutral density filters the beam intensity dynamic range is extended to 3000 , starting at a circulating current of $5 \mu \mathrm{A}$ with an integration time of $20 \mathrm{~ms}$, a wavelength filter centred around $450 \mathrm{~nm}$ and present beam sizes.

\section{DIFFRACTION AND DEPTH OF FIELD CONTRIBUTIONS TO THE MEASURED PROFILES}

In order to calculate the beam size from the measured light spot, it is necessary to evaluate the broadening introduced by the depth of field and the diffraction.

The depth of field is limited by a horizontal slit of width $w$ located in the focal plane of the focusing spherical mirror [1]. This slit is the main origin of diffraction in the horizontal plane. In the vertical plane, the only diffraction to be considered is that originating from the small aperture of the SR. The various contributions have been measured by varying the slit width $w$ and the wavelength $\lambda$ used. The evolution of the horizontal and vertical rms beam sizes as a function of the slit width $w$ are given in Fig. 1.

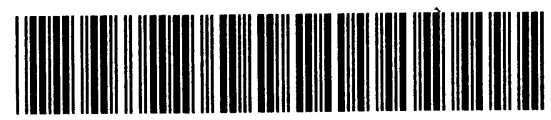




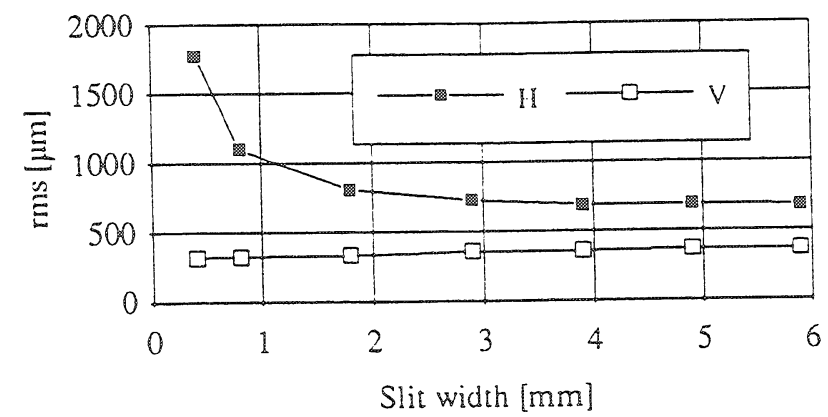

Fig. 1: Evolution of the horizontal and vertical rms beam sizes as a function of slit width $w$ at $450 \mathrm{~nm}$.

The vertical rms size of the light spot decreases slowly with the slit width $w$, whereas in the horizontal plane the size passes through a minimum betore increasing sharply for low values of $w$. This is due to the combination of depth of ficld and diffraction acting in opposite ways. An operational slit width of $2 \mathrm{~mm}$ has been chosen as an acceptable compromisc.

The natural diffraction contribution has been evaluated by measuring the vertical size of a beam at four different wavelengths, i.e. 450,622 and $800 \mathrm{~nm}$ with the $C C D$ and $254 \mathrm{~nm}$ with the intensifier coupled to the CCD: Fig. 2. A fifth point is given by a solar blind filter.

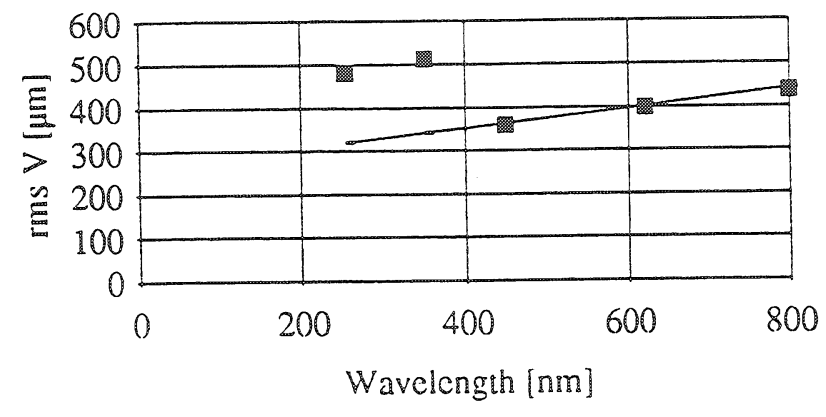

Fig. 2: Vertical light spot sizes measured at various wavelengths together with the expected spot size curve.

The spot size decreases with the wavelength as expected until the CCD is replaced by the intensifier, showing the additional spot broadening introduced by the intensifier. This broadening has been estimated to be $72 \mu \mathrm{m} \mathrm{rms}$ at the detector level, i.e. $360 \mu \mathrm{m}$ at the beam level.

Correction coefficients for estimating the beam size have been calculated from these measurements. The rms contributions, expressed in $\mu \mathrm{m}$, of the diffraction, $\sigma_{D H}$ and $\sigma_{D V}$, and of the longitudinal acceptance $\sigma_{L \Lambda}$ can be expressed as:

$$
\begin{aligned}
& \sigma_{D H}=1.45 \lambda / \mathrm{w} \\
& \sigma_{\mathrm{DV}}=3.9 \lambda^{2 / 3} \\
& \sigma_{\mathrm{LA}}=40 \mathrm{w}
\end{aligned}
$$

where the slit size $w$ is expressed in $\mathrm{mm}$ and the wavelength $\lambda$ in $\mathrm{nm}$. These contributions are subtracted quadratically from the measured light spot rms size. The main difficulty in measuring these correction coefficients is to have sufficient stable beam conditions. The broadening introduced by the gated intensifier-wavelength shifter is too large to permit precision beam size measurements. The calibration curve of the vertical light spot size versus emittance at the nominal operating parameters is given in Fig. 3.

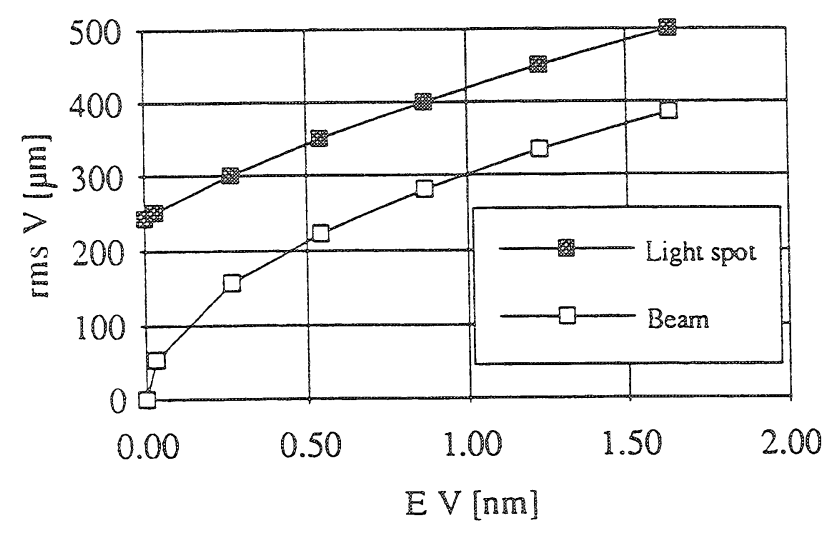

Fig. 3: Vertical Light spot size versus Emittance at zero dispersion: for $\beta y=91 \mathrm{~m}, \lambda=450 \mathrm{~nm}, w=2 \mathrm{~mm}$

A similar curve can be established for the horizontal case. As can be seen from this calibration curve, it is desirable to lower the wavelength at which the measurements are made for evaluating the present small beam emittances.

\section{OBSERVATIONS AND EMITTANCE ESTIMATIONS \\ A feature which became apparent during the previous} measurements were small beam size excursions. By varying the CCD integration time from 2 to $30 \mathrm{~ms}$ with a stable beam, the variation of the spot sizes could be measured: Fig. 4.

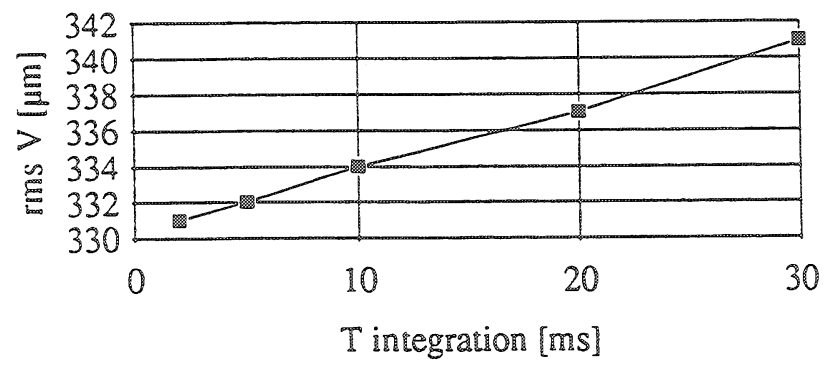

Fig. 4: Light spot size variations as a function of $C C D$ integration time.

Variations of $4 \mu \mathrm{m}$ of the horizontal projection and of $10 \mu \mathrm{m}$ of the vertical projection, at beam level, were recorded. Unfortunately the integration time could not be increased beyond $30 \mathrm{~ms}$ to characterise more precisely the frequency of the phenomenon. Neither was it possible to get precise 
enough information from the gated detcctor. The influence of these oscillations on estimated beam sizes is negligible in the horizontal plane but results in an enlargement of up to $2 \%$ in the vertical plane for a $20 \mathrm{~ms}$ integration time. This "wobbling" has been observed also with the pick-ups [2] and is suspected to be of $50 \mathrm{~Hz}$ frequency and be caused by a power cable. This particular effect should disappear in 1993. Nevertheless the possibility to increase the integration period beyond $40 \mathrm{~ms}$, actually up to $512 \mathrm{~ms}$, in order to study similar phenomena will be implemented.

During the first two years of running, the LEP emittances were close to the original design values of 42 and $1.7 \mathrm{~nm}$ and the emittances provided by the telescopes were consistent with the experimental Luminosity figures. In 1993 a new optics with a $90^{\circ}$ phase advance in both planes was introduced, resulting in much lower cmittances, typically 12 and $0.5 \mathrm{~nm}$. Despite the improvements implemented, the emitance figures provided by the telescopes for colliding beams were generally higher, sometimes up to $40 \%$, w.r.t the Luminosity figures. Several phenomena can be considered to explain it. The most likely ones are the lack of knowledge of the machinc optical functions at the telescopes, beam instabilities during the intcgration period, and magnetic coupling resulting in beam tilis which give larger apparent vertical emittances in the detector frame.

Precision cross-calibrations with the wire scanners have been started [3]. Due to the different opcrating conditions and locations of the two monitors, the results of these tests are delicate to interpret. Beam tilts and limited knowledge of the machine optical functions increase the difficulty of these comparisons. A lew good comparisons were made and are encouraging for the future. An upper limit on the precision of $+/-0.5 \mathrm{~nm}$ on the horizontal emittance and $+1-0.2 \mathrm{~nm}$ on the vertical emittance could be deduced. The relative accuracy is much better. Beam size changes of $5 \mu \mathrm{m}$ can be detected, see Fig. 4, and hence emituance changes of $2 \%$ are detectable with stable bcams. Systematic comparisons with the $X$-ray vertical profile monitors [4] are also lorescen in 1993.

The facilities with the fast shutuer were litule used. This is mainly due to the additional enlargement of the spot size and to the late availability of display software for the control room workstations. This mode is useful to compare the sizes of the different bunches and to observe fast turn by turn beam instabilities with the "burst" mode [1]. The new display facilities show centre of gravity and beam size evolution over the eight acquired profiles, taken at intervals from one to $256 \mathrm{LEP}$ tums.

As some measurements have indicated that the beam tails were not gaussian, preliminary tests to sudy these tails have been made by directing the beam light spot towards the edge of the CCD detector in order to increase its apparent dynamic range up to $1 / 10^{5}$. These tests will be continued.

The TV monitors with superimposed measurement results [1] are still the most used facility. For helping with the tuning of LEP, an eight bit lrame digitiser with fast $(40 \mathrm{~ms})$ hardware beam size calculations was installed. The results are averaged over ten measurements for noise rejection and displayed on a colour TV monitor in the control room: Fig. 5.

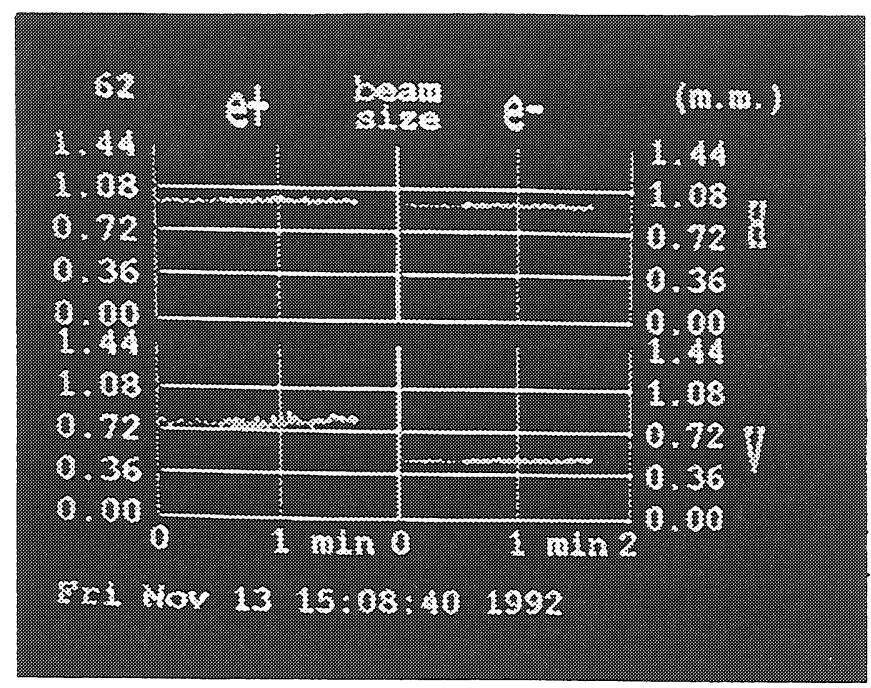

Fig. 5: Fast beam size display showing the evolution of the two beams over two minutes. It is updated every $600 \mathrm{~ms}$.

\section{PLANS FOR THE FUTURE}

For 1993 it is foreseen to install:

- a phosphor coated CCD sensitive down to $200 \mathrm{~nm}$ to improve the precision of the beam size measurement,

- a set of linite widh density filters for implementing a "corona"-type set-up to study the beam tails,

- a linear density filter set to keep automatically the beam signal close to its maximum during a whole run, - to have all deteciors equipped with Peltier cooling cells.

The system will be using a 68030 CPU running under OS 9 and directly connected to Ethernet which will increase the processing speed and suppress a layer of the original control set-up.

\section{ACKNOWLEDGEMENTS}

Acknowledgements are due to J.P. Bindi who designed and installed the telescope cooling with Peltier cells, to L. Brunel for the CCD measurements, to G.F. Ferioli and J. Provost who provided the hardware for the fast video acquisition and display of the beam sizes, to A. Bums for the video display software, and to E. Hatziangeli for the workstations software.

\section{REFERENCES}

[1] C. Bovet et al.: The LEP synchrotron light monitors, Proc. of the 1991 IEEE Acc. Conf., San Francisco

[2] J. Borer et al. : Harmonic analysis of coherent bunch oscillations in LEP, Proc. of the 1992 EPAC, Berlin

[3] J. Camas et al.: High resolution measurements of profiles with the LEP wire scanners, these proceedings.

[4] E. Rossa et al:: Measurement of vertical emittance at LEP from hard $X$-rays, these proceedings 